\title{
Electrochemical DNA Detection Methods to Measure Circulating Tumour DNA for Enhanced Diagnosis and Monitoring of Cancer ${ }^{\dagger}$
}

\author{
Bukola Attoye 1,*, Matthew Baker ${ }^{2}$, Chantevy Pou ${ }^{3}$, Fiona Thomson ${ }^{3}$ and Damion K. Corrigan 1 \\ 1 Department of Biomedical Engineering, University of Strathclyde, 40 George Street, Glasgow G1 1QE, UK; \\ damion.corrigan@strath.ac.uk \\ 2 Department of Pure and Applied Chemistry, University of Strathclyde, Technology and Innovation Centre, \\ 99 George Street, Glasgow G1 1RD, UK; matthew.baker@strath.ac.uk \\ 3 Wolfson Wohl Cancer Research Centre, Institute of Cancer Sciences, University of Glasgow, \\ Glasgow G61 1QH, UK; chantevy.pou@glasgow.ac.uk (C.P.); fiona.thomson@glasgow.ac.uk (F.T.) \\ * Correspondence: bukola.omolaiye@strath.ac.uk \\ † Presented at the 1st International Electronic Conference on Biosensors, 2-17 November 2020; \\ Available online: https://iecb2020.sciforum.net/.
}

Published: 2 November 2020

\begin{abstract}
Liquid biopsies are becoming increasingly important as a potential replacement for existing biopsy procedures which can be invasive, painful and compromised by tumour heterogeneity. This paper reports a simple electrochemical approach tailored towards point-of-care cancer detection and treatment monitoring from biofluids using a label-free detection strategy. The mutations under test were the KRAS G12D and G13D mutations, which are both important in the development and progression of many human cancers and which have a presence that correlates with poor outcomes. These common circulating tumour markers were investigated in clinical samples and amplified by standard and specialist PCR methodologies for subsequent electrochemical detection. Following pre-treatment of the sensor to present a clean surface, DNA probes developed specifically for detection of the KRAS G12D and G13D mutations were immobilized onto low-cost carbon electrodes using diazonium chemistry and 1-ethyl-3-(3dimethylaminopropyl) carbodiimide hydrochloride/N-hydroxysuccinimide coupling. Following the functionalisation of the sensor, it was possible to sensitively and specifically detect a mutant KRAS G13D PCR product against a background of wild-type KRAS DNA from the representative cancer sample. Our findings give rise to the basis of a simple and very low-cost system for measuring ctDNA biomarkers in patient samples. The current time to result of the system was $3.5 \mathrm{~h}$ with considerable scope for optimisation, and it already compares favourably to the UK National Health Service biopsy service where patients can wait weeks for their result. This paper reports the technical developments we made in the production of consistent carbon surfaces for functionalisation, assay performance data for KRAS G13D and detection of PCR amplicons under ambient conditions.
\end{abstract}

Keywords: electrochemical; DNA biosensors; KRAS; liquid biopsy; cancer point-of-care diagnostic tests

\section{Introduction}

Cancer is a genetic disease by nature, caused by mutations in certain genes thereby resulting in cellular malfunction [1]. Imaging tests can sometimes be inconclusive and generally do not provide information on the stage or type of cancer, so further biopsy is needed [2]. Collecting multiple biopsies from different regions of a primary tumour and associated metastasis is invasive and may 
pose serious medical risks [3]. The time point at which a tumour manifests clinical symptoms often correlates with the later stages of progression (e.g., Phases III and IV), when surgery and therapy are less effective because of, for example, metastasis or tumour cells acquiring a critical mass of mutations [4]. Furthermore, for some patients, surgical biopsy procedures are not possible; therefore, liquid biopsies that detect the presence of tumour DNA in blood hold promise as a non-invasive alternative.

Short fragments of cell-free DNA (cfDNA) are detectable in most body fluids including blood. In cancer patients, a fraction of cfDNA called circulating tumour DNA (ctDNA) can be found, which originates from tumours and may carry the same mutations and genetic alterations as those present in the primary tumour [5]. Although circulating tumour cells (CTCs) that have been shed into the vasculature of a primary tumour are carried around the body in circulation, they are present at levels of approximately 10 cells $/ \mathrm{mL}$ of blood, indicating that only very low concentrations are present in clinical samples. Compared to ctDNA, CTCs are rare in peripheral blood and are difficult to separate from other cells, adding further credibility to the use of ctDNA for liquid biopsy applications. Although the mechanism of ctDNA release from tumour cells is poorly understood [6], it is thought to be released in small quantities following apoptosis or necrosis. Circulating tumour DNA usually comprises $0.01-1 \%$ of the circulating free DNA in blood [7], and it is important to note that this can be shed as both single-stranded and double-stranded DNA [8]. Circulating tumour DNA can currently be detected in blood and other body fluids like lymph, urine, and stool [9]. Due to the small fraction of ctDNA masked in by large background levels of wild-type cell-free DNA, highly sensitive amplification reactions, such as polymerase chain reaction (PCR), will need to be employed to achieve discrimination. It is possible that a Point of care (PoC) measurement of circulating tumour DNA (ctDNA) may present a more practical means of detecting the presence of a tumour as well as capturing tumour heterogeneity, evaluating a response to treatment and monitoring disease recurrence $[9,10]$.

Kirsten rat sarcoma (V-Ki-ras) commonly abbreviated KRAS, is a member of the RAS family of proteins which are a part of at least six signalling pathways in a healthy human. V-Ki-ras Kirsten rat sarcoma is the most frequently mutated protein in human tumours with KRAS G12D and G13D being specific variants found across many tumour types including pancreatic, colorectal, non-small cell lung, and ovarian cancer [10]. V-Ki-ras Kirsten rat sarcoma activated mutations drive cancer initiation, progression, and drug resistance, directly leading to nearly a million deaths per year. VKi-ras Kirsten rat sarcoma mutations take place in approximately $90 \%$ of pancreatic cancers [11], 30\% of lung cancers [12], $60 \%$ of thyroid cancers, and $43 \%$ of colorectal cancers [13]. The topic of liquid biopsies is one such area where innovations in biomarker detection will enhance clinical outcomes for patients [14,15].

Electrochemical DNA biosensors represent an exciting approach to the fast, low-cost detection of clinically important biomarkers [16] at the point of care [17]. Electrochemical biosensors are used to directly convert a biological binding event to an electronic signal [18]. A range of electrode materials and electrochemical measurement approaches have been employed for sensitive measurements (e.g., cyclic voltammetry [19], differential pulse voltammetry [20], square wave voltammetry [21], and electrochemical impedance spectroscopy [22]). The potential of electrochemical biosensors once matured as a technology to provide efficient clinical workflows is immense. In DNA biosensing, a change in signal is obtained when recognition and hybridisation of two opposing strands of DNA occur as a result of their base-pair complementarity. A doublestranded DNA sequence with tumour-specific mutations can indicate the diagnosis of a specific cancer [11]. As the concentration of ctDNA increases when patients have advanced cancers, achieving high sensitivity for the DNA sensor is important for the early detection of disease and developing tailored therapies. Carbon electrodes are chemically inert, particularly at negative potential ranges in all media making them highly suitable electrode sensors for electroanalytical chemistry and giving them an advantage over metal electrodes [23]. Screen-printed carbon electrodes (SPCEs) are homogenous, simple, sensitive, cost-effective ( $\sim £ 2$ each) and disposable, making them preferable for rapid electrochemical analyses and suitable as electrodes for characterising the processes we 
employed, which were cyclic voltammetry (CV) and differential pulse voltammetry (DPV), two routinely used electrochemical measurement techniques.

Cyclic voltammetry and DPV are common analytical techniques that supply information on electron transfer reaction kinetics of any combined chemical reaction [24]. In both techniques, a potential waveform is applied to the working electrode (WE). The peak current obtained is directly influenced by hybridisation between the target and the immobilised probe DNA strands [25]. Both DPV and CV are useful for studying electro-activity and characterising the reductive and oxidative properties of compounds in solution. This work presents a KRAS G12D and G13D DNA oligonucleotide probe-modified sensor array that can accurately detect mutant KRAS amplicons and, therefore, forming the basis of a system for the accurate detection of ctDNA in patient samples and monitoring responses during treatment. This was achieved by amplifying mutant DNA isolated from human cancer cell lines recovered from clinical samples, using electrochemical techniques and SPCEs to detect a clinically relevant mutation, comparing the signal change from DNA hybridisation experiments involving wild-type KRAS and amplified KRAS mutant samples, varying concentrations of amplified products to determine concentration effects and establishing a limit of detection for the DNA amplification reaction. In this study, both DPV and CV were used depending on whether electrodes needed to be cleaned, electrografted or characterised during sensor measurement. Different voltage and scan ranges were selected for the measurements carried out. Due to the simplicity of the approach presented herein and the choice of steps employed in the assay, the system can be very easily automated and integrated into a final device capable of fast and seamless clinical measurements.

\section{Materials and Methods}

\subsection{Reagents}

Droplet digital PCR (ddPCR) assays, Supermix for probes, DG8TM cartridges and Droplet Generation Oil were obtained from Bio-Rad Laboratories Ltd., Hertfordshire, UK. Deionized water, sodium chloride, phosphate-buffered saline (PBS), sodium nitrate, 4-aminobenzoic acid, hydrochloric acid, ethanolamine, 2-(N-morpholino) ethanesulfonic acid (MES), 1-ethyl-3-(3dimethylaminopropyl) carbodiimide hydrochloride (EDC), N-hydroxysuccinimide (NHS), potassium ferricyanide, potassium chloride and potassium ferrocyanide were all purchased from Sigma-Aldrich, (Dorset, UK). Two hundred and fifty units of HotStarTaq Plus and dNTP Mix, PCR Grade $(200 \mu \mathrm{L})$, were purchased from Qiagen, (Manchester, UK). Phusion Direct PCR kit was purchased from thermo fisher scientific, (Renfrew, UK).

\subsection{Electrochemical Setup}

Screen-printed multi-carbon electrodes (DRP 8W110), as shown in Figure 1A below, were obtained from DropSens (Oviedo, Spain) with chip dimensions of $50 \times 27 \times 1 \mathrm{~mm}(\mathrm{~L} \times \mathrm{W} \times \mathrm{D})$. The chip contained eight carbon working electrodes with diameters of $2.95 \mathrm{~mm}$ and a carbon counter and silver reference electrode. The screen-printed fabrication process was specified by the manufacturers. 
(A)

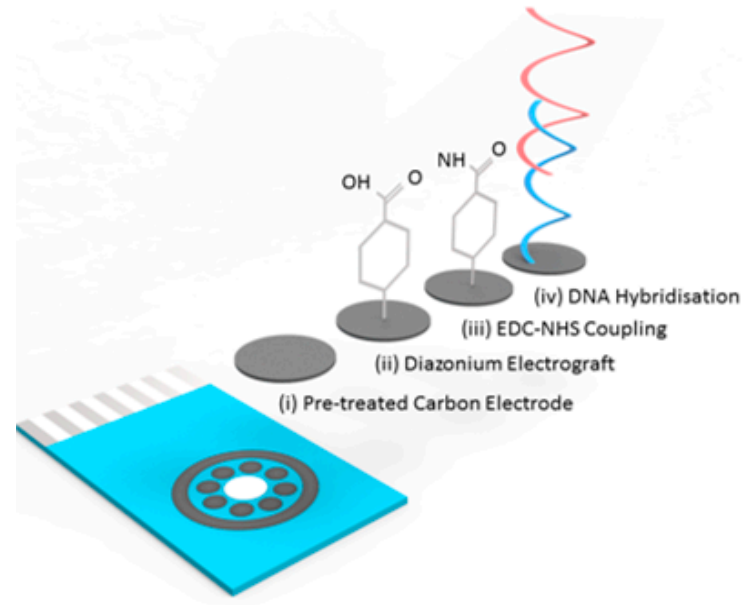

(B)

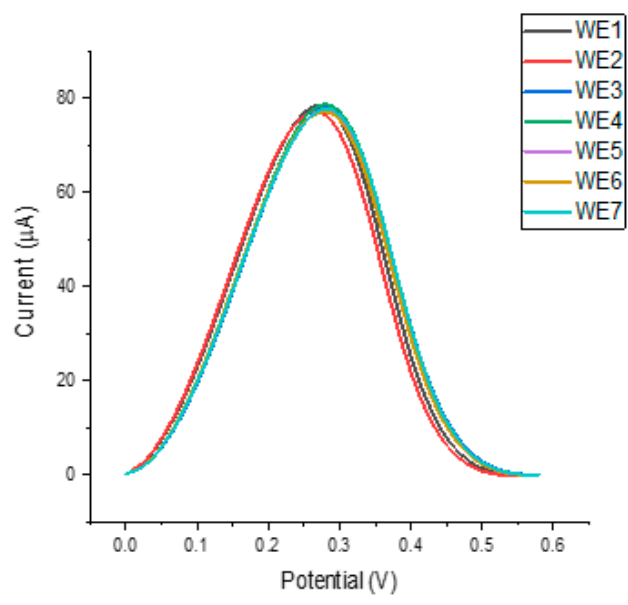

Figure 1. (A) Image of a screen-printed electrode array employing eight working electrodes with a common Ag reference and carbon counter electrodes along with a schematic showing DNA functionalisation and DNA target binding. (B) Differential pulse voltammetry (DPV) results showing repeated cycling of the eight working electrodes prior to modification and DNA introduction using the same current and voltage inputs.

\subsection{Electrochemical Measurements and Surface Functionalisation}

DNA hybridisation experiments were performed using a covalently attached layer of singlestranded DNA probes. The surface functionalisation protocol is illustrated in Figure 1A. To prepare the surface of the carbon electrodes for DNA probe attachment, it was necessary to first use a surface pre-treatment by applying $1.4 \mathrm{~V}$ for $1 \mathrm{~min}$ in $0.5 \mathrm{M}$ acetate buffer solution (ABS) containing $20 \mathrm{mM}$ $\mathrm{NaCl} 8$ (pH 4.8) via CV. Next, $2 \mathrm{mM} \mathrm{NaNO}_{2}$ solution with $2 \mathrm{mM} 4$-aminobenzoic acid was prepared in $0.5 \mathrm{M} \mathrm{HCl}$ and stirred for approximately $5 \mathrm{~min}$ at room temperature to produce a diazonium compound. The activated diazonium solution was then scanned using $\mathrm{CV}$ from +0.4 to $-0.6 \mathrm{~V}$ at a scan rate of $100 \mathrm{mV} / \mathrm{s}$ followed by a wash with deionised (DI) water. The resulting 4-carboxyphenyl (AP) film was activated on the electrode's surface with $100 \mathrm{mM}$ EDC and $20 \mathrm{mM}$ NHS in $100 \mathrm{mM}$ MES buffer ( $\mathrm{pH}$ 5.0) for $60 \mathrm{~min}$ to form an ester that allowed for efficient conjugation to the aminemodified ssDNA probe. Ferricyanide buffer $(5 \mu \mathrm{M})$ was used to characterise the sensor surface. Unless specifically stated, all the reported steps and measurements were carried out at room temperature.

\subsection{Genomic DNA Sample Preparation, DNA Probe Design, and Sample Amplification}

For assay development, copies of the KRAS pG13D mutant and wild-type DNA were amplified from genomic DNA (gDNA) isolated from SK-UT-1 cells. Levels of both mutant and wild-type DNA were determined using ddPCR assays (KRAS WT for p.G13D c.35G >A, assay ID dHsaCP2000002) in combination with a QX200TM Droplet DigitalTM PCR system (Bio-Rad Laboratories Ltd., Hertfordshire, UK) following the manufacturer's instructions. Briefly, 5-10 ng of gDNA isolated from SK-UT-1 cells was mixed with ddPCR Supermix for probes (N0 dUTP) and fluorescein amidite (FAM)-labelled KRAS p.G13D primers/probe and hexachloro-fluorescein 9 (HEX)-labelled KRAS WT primers/probes in the presence of a restriction enzyme and in a volume of $20 \mu \mathrm{L}$. Reaction samples were loaded onto a DG8TM cartridge with $70 \mu \mathrm{L}$ of droplet generation oil for Probes according to the Droplet Generator Instruction Manual (Bio-Rad Laboratories Ltd., Hertfordshire, UK). The PCR cycling conditions for the generated droplets were as follows: initial enzyme activation at $95^{\circ} \mathrm{C}$ for $10 \mathrm{~min}$, followed by 40 cycles of denaturation at $94^{\circ} \mathrm{C}$ for $30 \mathrm{~s}$, and annealing/extension at $55^{\circ} \mathrm{C}$ for 1 min, after which it ended with a final enzyme deactivation at $98^{\circ} \mathrm{C}$ for $10 \mathrm{~min}$. Data acquisition after thermal cycling was performed using the QX200 Droplet Reader and the QuantaSoft Software (BioRad Laboratories Ltd., Hertfordshire, UK). 
The design of the PCR primers and probes used in this study was based on the published sequence of KRAS pG12D and pG13D under accession number NC_000012.12 ([26] Amine-modified synthetic oligonucleotides (KRAS G13D) designed, as shown in Table 1 below, with a concentration of $200 \mu \mathrm{M}$ was obtained from Sigma-Aldrich, $\mathrm{UK}$, and stored at $-80^{\circ} \mathrm{C}$ prior to aliquoting for use as probes. A wild-type probe (without the single base mutation) was also designed for use as a negative control. The DNA probe stocks were diluted to a concentration of $2 \mu \mathrm{M}$ in $0.1 \times$ PBS prior to immobilisation. Primer-BLAST software was used to design the PCR primers employed in this study. The forward primers had a GC content of $55 \%$ while the reverse primer had a GC content of $39.13 \%$ with an estimated product length of 88 with low self-complementarity.

Table 1. List of DNA sequences employed in this study.

\begin{tabular}{cc}
\hline \multicolumn{2}{c}{ KRAS G13D Probe and Primer Sequences } \\
\hline 23 Bases Wild-Type Hybridisation Probe & TGGAGCTGGTGGCGTAGGCAAGA \\
23 Bases Mutant Hybridisation Probe & TGGAGCTGGTGACGTAGGCAAGA \\
Forward Primer (Wild-Type) & TGTGGTAGTTGGAGCTGGTG \\
Forward Primer (Mutant) & TGTGGTAGTTGGAGCTGATG \\
PCR Probe (Mutant) & TCTTGCCTACGCCACCAGCTCCA \\
Reverse Primer & TTGTGGACGAATATGATCCAACA \\
\hline
\end{tabular}

For PCR amplification, samples of extracted wild-type and KRAS G13D-mutated DNA was amplified using the Phusion Direct PCR kit following the protocol and reaction set-up guide outlined by Thermo Scientific, UK. Phusion Blood II DNA polymerase $(1 \mu \mathrm{L}), 2 x$ PCR Buffer $(25 \mu \mathrm{L}), 50 \mathrm{mM}$ EDTA $(2.5 \mu \mathrm{L}), 50 \mathrm{mM} \mathrm{MgCl} 2$ solution $(1.5 \mu \mathrm{L})$ and $100 \%$ DMSO $(2.5 \mu \mathrm{L})$ were all included in the reaction mix and dispensed into appropriate PCR tubes. One microlitre of Template DNA, $5 \mu \mathrm{L}$ forward and reverse primers and $10 \mu \mathrm{L}$ ultrapure water were added to the master PCR tube containing the reaction mix, and the thermal cycler was programmed to start with an initial heatactivation step at $98^{\circ} \mathrm{C}$ for $300 \mathrm{~s}$. Temperature specifications for denaturing, annealing and extending were set at $90 \mathrm{~s}$ for $94^{\circ} \mathrm{C}, 65^{\circ} \mathrm{C}$ and $72{ }^{\circ} \mathrm{C}$, respectively. A final extension for $60 \mathrm{~s}$ at $72{ }^{\circ} \mathrm{C}$ was set, and the PCR conditions were set for 37 cycles. The PCR amplification of wild-type and KRAS G13D samples was performed using the miniPCR thermal cycler [27], and amplicon concentrations were confirmed using the Qubit 4 fluorometer and dsDNA broad range quantification assay [1].

\section{Results and Discussion}

\subsection{Assay Workflow and Development}

The use of an SPCE with multiple working electrodes allows each electrode to be individually modified whilst rapidly carrying out simultaneous measurements of peak currents and charge transfer resistance. The electrode functionalisation process is summarised in Figure 1A. Electrografting using in situ generated diazonium cations is important for modifying the surface of the SPCE by allowing the formation of covalent bonds between the carbon surface and organic films. The EDC is an established zero-length cross-linking agent that has been employed in coupling carboxyl groups to primary amines in various applications [28]. One of the main benefits of EDC coupling is its water solubility that allows direct bioconjugation without prior organic solvent dissolution. To increase the stability of the active ester, NHS was introduced to modify the aminereactive chemical substance by converting it to an active NHS ester, thus maximising the efficiency of the EDC-mediated coupling reactions. The reproducibility of this hybridisation sensor was explored by simultaneously analysing all eight Wes from the multielectrode after the surface modification steps. Figure 1B shows a high level of consistency in the peak height, potential and width. The multiplexed analysis we employed greatly reduced the analysis time because of the high throughput of samples and minimised reagent consumption. After introducing the probe solution to the surface of the modified electrodes, the remaining active groups on the electrode were blocked 
using ethanolamine to produce a consistent sensing layer in order to enable DNA specificity and stability in terms of the DNA binding response.

\subsection{DNA Sensor Hybridisation Specificity}

Having achieved a consistent behaviour of modified electrodes on the same chip, the next step was to test the assay's response to incubation in a representative KRAS sample using the designed probes. We explored the ability of the probe-modified electrodes to discriminate between G13D mutant and wild-type KRAS sequences in representative clinical samples. To investigate specificity levels and gain an initial impression of assay sensitivity, a series of electrodes were functionalised with KRAS G13D mutant and wild-type probe sequences. The results of these experiments are summarised in Figure 2A, which shows the percentage change in the $\mathrm{CV}$ peak current following target hybridisation. For macroscale electrodes functionalised with biological molecules, such as DNA or antibodies, the expectation is that differential pulse voltametric peak currents will reduce upon target hybridisation. It has been observed that these effects can be reversed when micro- or nanoscale electrodes are employed [17,30], but for this study, the electrodes employed were comfortably on the macro scale (diameter $=2.95 \mathrm{~mm}$ ). Therefore, we expected a reduction in the peak current when specific DNA hybridisation had taken place, and this was found to be the case for lower concentrations of DNA (pico-to-low nanomolar concentrations) as shown in Figure 2B,C. For nanomolar $(>10 \mathrm{nM})$ and micromolar concentrations, an increase in the peak current following hybridisation was consistently observed (and has also been observed in other data from our lab involving SPCEs) [31] for carbon electrodes which is likely explained by the high surface density of hybridised DNA amplicons changing the interfacial properties of the electrode and, therefore, altering the electrochemical response. The underlying physical mechanism of this effect is actively under investigation. Figure $2 \mathrm{~A}$ shows that when mutant and wild-type oligonucleotide probe sequences functionalised SPCEs were incubated in a representative sample containing the G13D mutation, there was hybridisation in both cases, the signal change was greater for the wildtype probe because of the high background of wild-type DNA and the comparatively low fraction of mutated KRAS G13D present in the representative clinical sample. Similar behaviour was observed for KRAS G12D probe functionalised electrodes for a representative sample for that particular mutation, showing the wild-type KRAS DNA hybridised strongly to the nucleic acid modified carbon surfaces. As a result of these findings and the inability to electrochemically discriminate between positive and negative samples owing to the strong influence of background DNA in the sample, DNA amplification strategies were developed and tested in order to ensure the production of unequivocal detection of ctDNA mutations. 
(A)

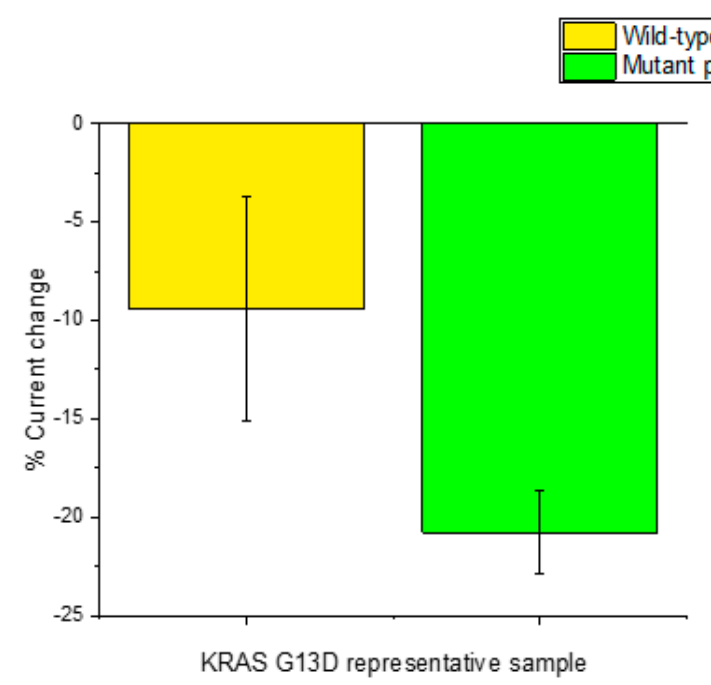

(B)

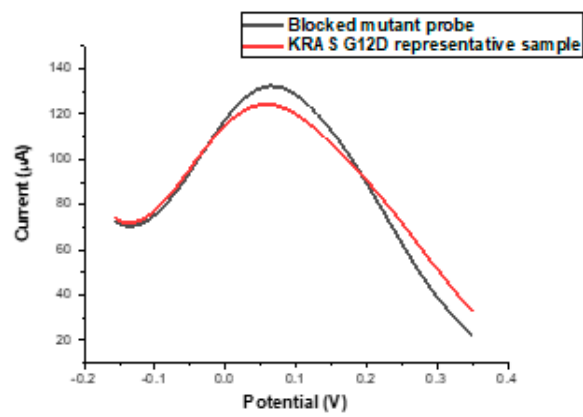

(C)

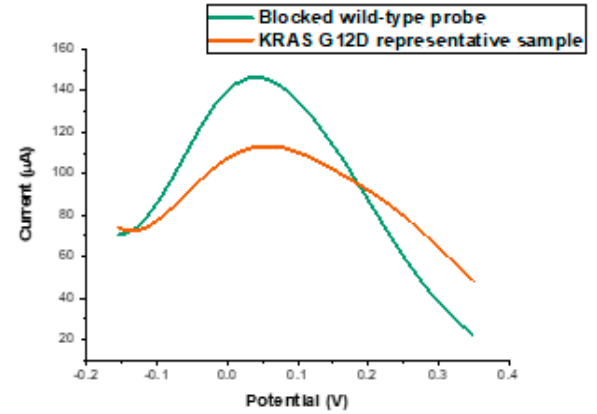

Figure 2. (A) CV percentage signal change in response to mutant and wild-type probes hybridized with genomic KRAS G13D ssDNA. (B,C) DPV signal changes in response to incubated KRAS G12D ssDNA hybridised with mutant and wild-type (WT) oligonucleotides, respectively.

\subsection{KRAS G13D Amplification}

Primers used for the PCR amplification were tailored to selectively amplify the mutant target from a pool of mutant and wild-type sequences in a sample by varying the single nucleotide responsible for the mutation in the primer sequence. Adopting this approach allowed us to effectively enrich the number of mutated DNA sequences in the sample without amplifying the wild-type in order to produce a signal change above the background signal generated by the KRAS wild-type DNA non-specifically associating with the oligonucleotide probe sequences for KRAS G13D.

In selecting the approach reported here, ctDNA detection could potentially be coupled to a DNA amplification reaction, because it allows the possibility of developing a multiplexed panel of DNA sequences on a single chip, meaning that commonly mutated genes could all be identified in parallel (e.g., KRAS, TP53, BRCA1/2, IDH-1). This approach of developing biomarker "panels" is thought to be one of the key advantages of this approach [32].

From Figure 3, when the wild-type probe-sequence modified electrodes were hybridised with KRAS G13D amplicons, alterations in the peak current were not observed, indicating no significant hybridisation. The mutant amplicons when incubated with mutant probe modified electrodes gave rise to a very significant signal change $(\sim 350 \%)$, indicating hybridisation with highly concentrated DNA samples (nano-micromolar concentration ranges) because of the strong positive signal change. Subsequent UV-Vis measurements on the amplicon confirmed this to be the case with estimated amplicons concentrations being in the range of 250-500 $\mu \mathrm{M}$. These findings were highly satisfying, i.e., that the surface-tethered KRAS G13D mutant probe sequence could, in fact, discriminate between the mutant and wild-type samples based on the presence or absence of PCR amplicons for KRAS G13D high sensitivity. This in fact represented a sort of double specificity for the PCR-based assay, because the primer design had already been shown to specifically amplify the mutated sequence so coupling in the specificity of the electrochemical probe sequence meant that the assay would be able to successfully discriminate mutant amplicons from the sample. Having established the specificity of the assay and the nature of the electrochemical change, the next step involved verifying the sensitivity of the assay and dose-response effects for the KRAS G13D mutant PCR product. 


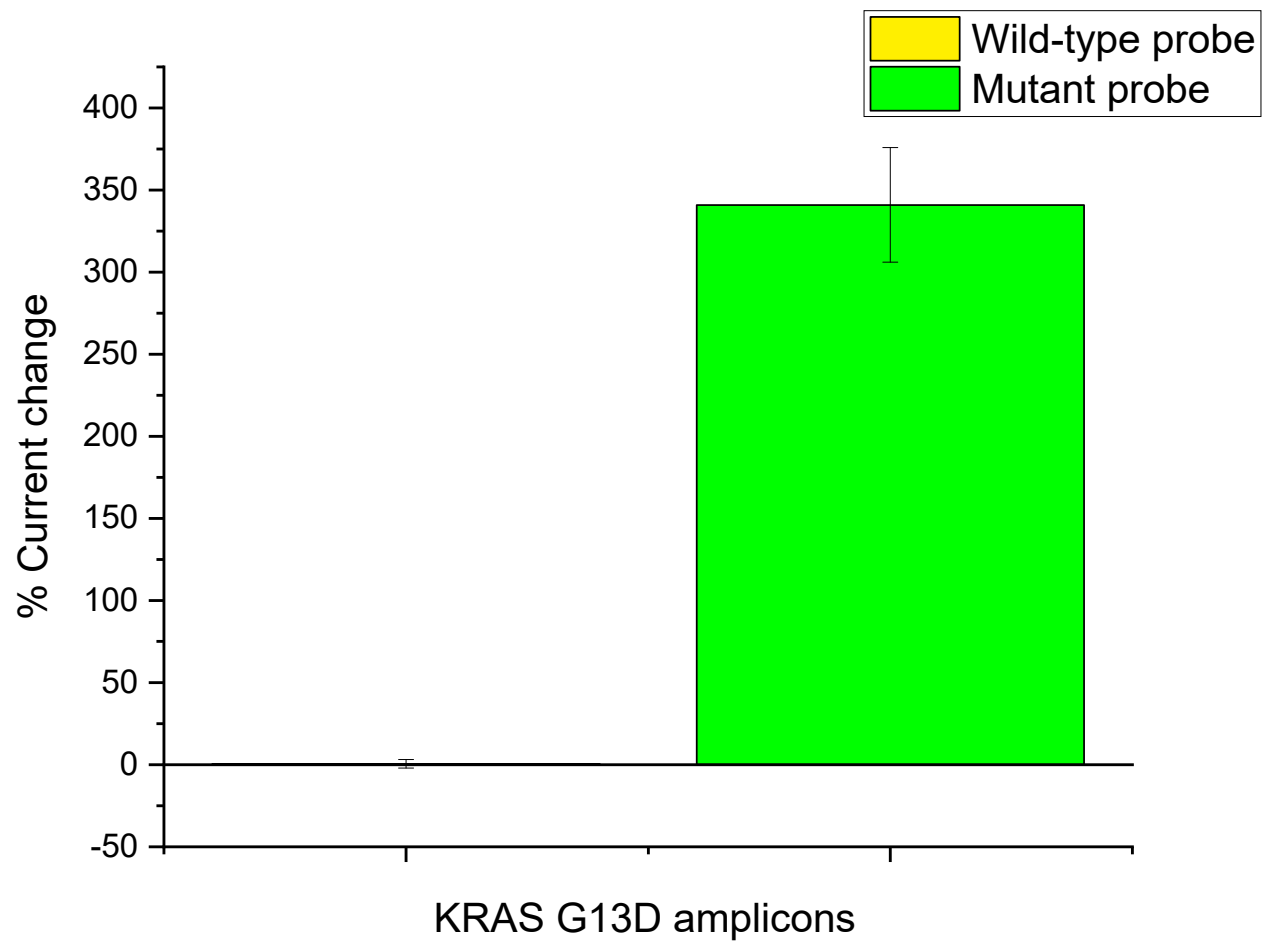

Figure 3. Successful amplification of KRAS G13D mutants further confirmed by a large percentage signal ratio between mutant probe and G13D mutant amplicons.

\subsection{Concentration Dose Response}

After establishing PCR primer specificity, ssDNA probe specificity and electrochemical signal changes in the correct direction and magnitude, it was important to investigate dose-response effects. In these experiments, non-amplified and amplified samples were diluted and a dose-response curve was constructed (see Figure 4A,B). For the unamplified sample (Figure 4A), the lowest concentration $(0.85 \mathrm{ng} / \mu \mathrm{L})$ showed the lowest reduction in oxidative peak current post-hybridisation with signal change increasing as sample concentration increased. The issue here, however, was the specificity of the probe-target interaction (as shown earlier) and the relatively small signal change brought about by incubation with unamplified samples. Owing to the fact that these were relatively low concentrations of DNA, leading to limited hybridisation, the signal changes were negative in direction, i.e., a decrease in the DPV peak current. On the other hand, the amplified sample produced a dose-response curve with higher signal changes which were positive in direction due to the specific enrichment of the mutant sequence concentration with smaller standard deviations because of the hybridisation of strands of similar lengths (the unamplified samples contained heterogenous strand lengths because it was unprocessed DNA) and, in effect, the full fraction of cfDNA from a patient. Achieving good sensitivity is very important as the concentration of circulating free DNA released by tumour cells is usually in proportion to the stage of cancer [33]. As circulating nucleic acids are present in blood at $\mathrm{ng} / \mathrm{mL}$ levels, which based on the fragment length is analogous to a picomolar concentration, a minimum of femtomolar sensitivity will be beneficial for detection of tumourspecific sequences [14]. Many published biosensor studies achieved such sensitivity levels through the use of exotic electrode modifications, typically involving the production of electrodes modified with graphene, nanoparticles, carbon nanotubes, etc. In our case, we opted to keep the electrode substrate low cost, easy to produce and coupled to a PCR reaction to achieve the desired sensitivity and specificity. Whilst our approach leads to a trade-off in terms of time to result, it ensures specific 
amplification and sensitive and specific hybridisation signals giving confidence in the result whilst achieving an overall time to result which is a significant improvement over the current clinical practice. The ctDNA concentration response shown in Figure 4 shows a clear dose-response effect which predicts that an increase in ctDNA, per unit concentration, will result in a larger electrochemical signal response in the positive direction (i.e., increasing DPV peak current). Since levels of ctDNA are strongly correlated with tumour stage and response to therapy [33,34], there is a clear potential for this system to be applied in measuring how a patient's cancer treatment is progressing.

(A)

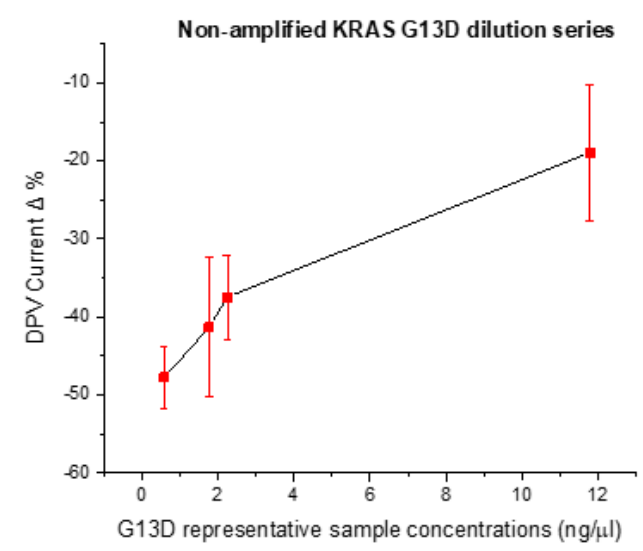

(B)

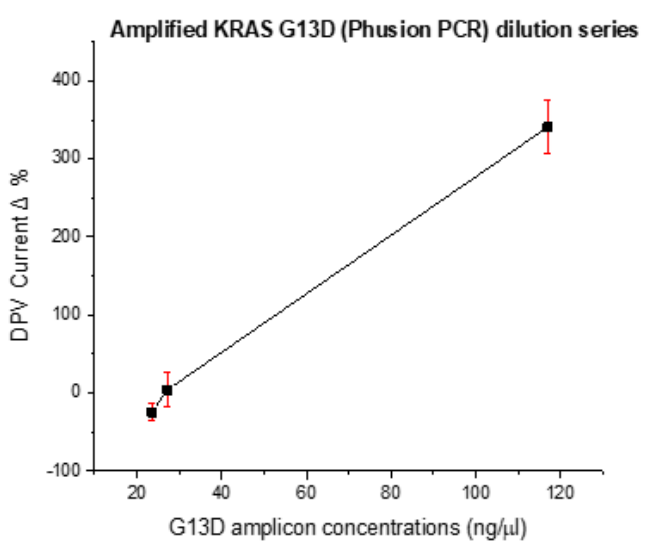

Figure 4. ctDNA response at different concentrations in a dilution series (A) DPV Peak currents percentage change for genomic KRAS G13D representative clinical sample at different concentrations (B) DPV Peak currents percentage change for KRAS G13D amplicons at different concentrations.

The findings of this study on ctDNA amplification are in agreement with several previous studies that were also able to successfully detect ctDNA KRAS mutations in patient samples using the ddPCR technique [34-37]. Electrochemical detection will quickly and accurately screen for cancer so treatment can be initiated as quickly as possible. In addition, this study shows that electrochemical sensors can be directly coupled to a standard PCR reaction that employs standard primers and reagents and does not require optimisation, meaning that amplification reactions for other ctDNA markers can be developed off-chip and transferred directly into the assay to produce a ctDNA panel.

The current time to result for cancer detection in a clinical setting is 2-3 days (including sample transportation) for a non-complicated biopsy analysis and 7-10 days for a complicated biopsy analysis [36]. In the UK, the National Health Service mutation typing following biopsy can take up to 9 weeks [37]. In total, the DNA isolation from blood, clean up and PCR amplification took approximately $150 \mathrm{~min}$. The ctDNA target incubation took approximately $60 \mathrm{~min}$, while the $\mathrm{CV}$ and DPV pre- and post-hybridisation measurements for each electrode took less than $10 \mathrm{~min}$. This gives a total of $3.5 \mathrm{~h}$. However, through optimisation and device integration, we believe there is considerable room for optimisation in terms of time to result. The current analysis time of $3.5 \mathrm{~h}$ is a big stride towards PoC provision for ctDNA profiling in a healthcare setting. This can be further optimised by isothermal amplification which can cut down the number of thermal cycles and, in turn, reduce the overall amplification time from $2 \mathrm{~h}$ to $1 \mathrm{~h}$ [38]. Near future work will explore the detection of other KRAS mutations and mutations in other genes involved in cancer, e.g., P53 and BRCA1. Analysing multiple mutations simultaneously in a given sample without prior knowledge of the alterations using multiplex techniques and direct detection of ctDNA from cancer patient samples will support the future direction of PoC clinical testing. 


\section{Conclusions}

A simple DNA sensor requiring no labelling processes or external indicators was developed using a multi-carbon electrode. We were able to design a PCR reaction capable of amplifying either mutant KRAS G13D or wild-type KRAS through primer choice from representative patient samples. In parallel, an electrochemical detection scheme involving a DNA hybridisation technique and screen-printed carbon electrodes were developed and shown through a series of comparative measurements to be sensitive and specific for the KRAS G13D mutation. Differential pulse voltammetry measurements provided the desired response and showed detection was possible from samples containing as few as $0.58 \mathrm{ng} / \mu \mathrm{L}$ amplicons. In addition, the response was found to be consistent with previously observed results, i.e., large signal decreases being evident upon amplification of the mutant allele, offering the promise of quantitation of mutant sequences from clinical samples. These results raise the prospect of simple, rapid and cost-effective measurement of nucleic acid tumour markers from blood and other body fluids. The current time to result of the electrochemical sensor was $3.5 \mathrm{~h}$, providing significant scope for optimisation. It is important to note that the sensor being developed can be potentially used for both early detection of cancer and monitoring the response to cancer treatment and, thus, increases the possibility of tailoring therapy and early diagnosis.

Author Contributions: Conceptualization, D.K.C.; methodology, B.A.; validation, D.K.C.; formal analysis, B.A.; investigation, B.A.; resources, F.T. and C.P.; data curation, B.A.; writing-original draft preparation, B.A.; writing - review and editing, B.A. and D.K.C.; supervision, D.K.C., and M.J.B.; project administration, B.A. and D.K.C. All authors have read and agreed to the published version of the manuscript.

Funding: The lead author was supported by EPSRC (grant reference number EP/L015595/1). F.T. and C.P. were supported by CRUK and ECMC core funding.

Acknowledgments: Special gratitude goes to CDT Medical Devices at the University of Strathclyde through which the grant was received.

Conflicts of Interest: The authors declare no conflict of interest. The funders had no role in the design of the study; in the collection, analyses, or interpretation of data; in the writing of the manuscript, or in the decision to publish the results.

\section{References}

1. National Cancer Institute. Genetic Changes and Cancer. In Genetic Changes and Cancer; 2019. Available online: https://www.cancer.gov/about-cancer/causes-prevention/genetics (accessed on 20 July 2019).

2. Othman, E.; Wang, J.; Sprague, B.L.; Rounds, T.; Ji, Y.; Herschorn, S.; Wood, M.E. Comparison of false positive rates for screening breast magnetic resonance imaging (MRI) in high risk women performed on stacked versus alternating schedules. Springerplus 2015, 4, 4-9.

3. Shyamala, K.; Girish, H.C.; Murgod, S. Risk of tumor cell seeding through biopsy and aspiration cytology. J. Int. Soc. Prev. Community Dent. 2014, 4, 5-11.

4. Holden, J. Diagnosing cancer in primary care. Bmj 2018, 320, 63-72.

5. Vendrell, J.A.; Taviaux, S.; Béganton, B.; Godreuil, S.; Audran, P.; Grand, D.; Clermont, E.; Serre, I.; Szablewski, V.; Coopman, P.; et al. Detection of known and novel ALK fusion transcripts in lung cancer patients using next-generation sequencing approaches. Sci. Rep. 2017, 7, 12510.

6. Gorgannezhad, L.; Umer, M.; Islam, N.; Nguyen, N.; Shiddiky, M.J.A. Lab on a Chip Circulating tumor DNA and liquid biopsy: Opportunities, challenges, and recent advances in detection technologies. Lab Chip 2018, 18, 1174-1196.

7. Chu, Y.; Cai, B.; Ma, Y.; Zhao, M.; Ye, Z.; Huang, J. Highly sensitive electrochemical detection of circulating tumor DNA based on thin-layer $\mathrm{MoS}_{2} /$ graphene composites. RSC Adv. 2016, 6, 22673-22678.

8. Schwarzenbach, H.; Hoon, D.S.B.; Pantel, K. Cell-free nucleic acids as biomarkers in cancer patients. Nat. Rev. Cancer 2011, 11, 426-437.

9. Taly, V.; Pekin, D.; Benhaim, L.; Kotsopoulos, S.K.; Le Corre, D.; Li, X.; Atochin, I.; Link, D.R.; Griffiths, A.D.; Pallier, K.; et al. Multiplex picodroplet digital PCR to detect KRAS mutations in circulating DNA from the plasma of colorectal cancer patients. Clin. Chem. 2013, 59, 1722-1731. 
10. Consortium, I. HHS Public Access. Cancer Discov. 2018, 7, 818-831.

11. Kulemann, B.; Kulemann B.; Rösch, S.; Seifert1, S.; Timme, S.; Bronsert, P.; Seifert, G.; Martini, V.; Kuvendjiska, J.; Glatz, T.; Hussung, S.; et al. Pancreatic cancer: Circulating Tumor Cells and Primary Tumors show Heterogeneous KRAS Mutations. Sci. Rep. 2017, 7, 4510.

12. Shackelford, R.E.; Whitling, N.A.; McNab, P.; Japa, S.; Coppola, D. KRAS Testing: A Tool for the Implementation of Personalized Medicine. Genes Cancer 2012, 3, 459-466.

13. Bettegowda, C.; Sausen, M.; Leary, R.J.; Kinde, I.; Wang, Y.; Agrawal, N.; Bartlett, B.R.; Wang, H.; Luber, B.; Alani, R.M.; et al. Detection of circulating tumor DNA in early- and late-stage human malignancies. Sci. Transl. Med. 2014, 6, 224ra24.

14. Kelley, S.O. What Are Clinically Relevant Levels of Cellular and Biomolecular Analytes? ACS Sensors 2017, 2, 193-197.

15. Crossley, L.; Attoye, B.; Vezza, V.; Blair, E.; Corrigan, D.K.; Hannah, S. Establishing a Field-Effect Transistor Sensor for the Detection of Mutations in the Tumour Protein 53 Gene (TP53)-An Electrochemical Optimisation Approach. Biosensors 2019, 9, 141.

16. Khanmohammadi, A.; Aghaie, A., Vahedi, E., Qazvini, A., Ghanei, M., Afkhami, A.; Bagheri, H. Electrochemical biosensors for the detection of lung cancer biomarkers: A review. Talanta 2019, 206, 120251.

17. Butterworth, A.; Ward, A.C. Analytical Methods Electrochemical detection of oxacillin resistance with SimpleStat: A low cost integrated potentiostat and sensor platform ${ }^{+}$. Anal. Methods 2019, 11, 1958-1965.

18. Grieshaber, E.R.D.; MacKenzie, R.; Voros, J. Electrochemical Biosensors-Sensor Principles and Architectures. Sensors 2008, 8, 1400-1458.

19. Shoaie, N.; Forouzandeh, M.; Omidfar, K. Voltammetric determination of the Escherichia coli DNA using a screen-printed carbon electrode modified with polyaniline and gold nanoparticles. Microchim. Acta 2018, 185, 1-9.

20. Butterworth, A.; Blues, E.; Williamson, P.; Cardona, M.; Gray, L. SAM Composition and Electrode Roughness Affect Performance of a DNA Biosensor for Antibiotic Resistance. Biosensors 2019, 9, 22.

21. Tsaloglou, M.-N.; Nemiroski, A.; Camci-Unal, G.; Christodouleas, D.C.; Murray, L.P.; Connelly, J.T.; Whitesides, G.M. Handheld isothermal amplification and electrochemical detection of DNA in resourcelimited settings. Anal. Biochem. 2018, 543, 116-121.

22. Li, P.Q.; Piper, A.; Schmueser, I.; Mount, A.R.; Corrigan, D.K. Correction: Impedimetric measurement of DNA-DNA hybridisation using microelectrodes with different radii for detection of methicillin resistant Staphylococcus aureus (MRSA). Analyst 2017, 142, 2849.

23. Yang, N.; Waldvogel, S.R.; Jiang, X. Electrochemistry of Carbon Dioxide on Carbon Electrodes. ACS Appl. Mater. Interfaces 2016, 8, 28357-28371.

24. Elgrishi, N.; Hammon, K.; McCarthy, B.; Eisenhart, T.; Dempsey, J. A Practical Beginner's Guide to Cyclic Voltammetry. J. Chem. Educ. 2017, 95, 197-206.

25. Rashid, J.I.A.; Yusof, N.A. The strategies of DNA immobilization and hybridization detection mechanism in the construction of electrochemical DNA sensor: A review. Sens. Bio-Sens. Res. 2017, 16, 19-31.

26. NCBI. 2020. Available online: https://www.ncbi.nlm.nih.gov/gene/3845 (accessed on 20 January 2020).

27. Amplyus. Bluegel-Electrophoresis. 2017. Available online: https://www.minipcr.com/ (accessed on 7 October 2019).

28. Obaje, E.A.; Cummins, G.; Schulze, H.; Mahmood, S.; Desmulliez, M.P.Y.; Bachmann, T.T. Carbon screenprinted electrodes on ceramic substrates for label-free molecular detection of antibiotic resistance. $J$. Interdiscip. Nanomedicine 2016, 1, 93-109.

29. Russell, C.; Ward, A.C.; Vezza, V.; Hoskisson, P.; Alcorn, D. Biosensors and Bioelectronics Development of a needle shaped microelectrode for electrochemical detection of the sepsis biomarker interleukin-6 (IL-6) in real time. Biosens. Bioelectron. 2019, 126, 806-814.

30. Manzanares-Palenzuela, C.L.; Fernandes, E.G.R.; Lobo-Castañón, M.J.; López-Ruiz, B.; Zucolotto, V. Impedance sensing of DNA hybridization onto nanostructured phthalocyanine-modified electrodes. Electrochim. Acta 2016, 221, 86-95.

31. Attoye, B.; Pou, C.; Blair, E.; Rinaldi, C.; Thomson, F.; Baker, M. J.; Corrigan, D.K. Electrochemical Sensor for the Detection of Circulating Tumour DNA in Human Fluids. Biosensors 2020, 10, 156.

32. Perakis, S.; Speicher, M.R. Emerging concepts in liquid biopsies. BMC Med. 2017, 15, 1-12.

33. Wang, X.; Wang, L.; Su, Y.; Yue, Z.; Xing, T. Plasma cell-Free DNA quantification is highly correlated to tumor burden in children with neuroblastoma. Cancer Med. 2018, 7, 3022-3030. 
34. Reece, M.; Saluja, H.; Hollington, P.; Karapetis, C.S.; Vatandoust, S.; Young, G.P.; Symonds, E.L. The Use of Circulating Tumor DNA to Monitor and Predict Response to Treatment in Colorectal Cancer. Front. Genet. 2019, 10, doi:10.3389/fgene.2019.01118.

35. DeMuth, C.; Spindler, K.-L.G.; Johansen, J.S.; Pallisgaard, N.; Nielsen, D.; Hoegdall, E.; Vittrup, B.; Sorensen, B.S. Measuring KRAS Mutations in Circulating Tumor DNA by Droplet Digital PCR and NextGeneration Sequencing. Transl. Oncol. 2018, 11, 1220-1224.

36. Coutinho, F.; Pancas, R.; Magalha, E.; Bernardo, E.; Antunes, M.J. Diagnostic value of surgical lung biopsy: Comparison with clinical and radiological diagnosis. Eur. J. Cardiothorac Surg. 2008, 33, 781-785.

37. NHS. Biopsy. 01.06.2018, 2018. Available online: https://www.nhs.uk/conditions/biopsy/ (accessed on 18 July 2019).

38. Chung, C.H.; Kim, J.H. One-step isothermal detection of multiple KRAS mutations by forming SNP specific hairpins on a gold nanoshell. Analyst 2018, 143, 3544-3548.

Publisher's Note: MDPI stays neutral with regard to jurisdictional claims in published maps and institutional affiliations.

(C) 2020 by the authors. Licensee MDPI, Basel, Switzerland. This article is an open access article distributed under the terms and conditions of the Creative Commons Attribution (CC BY) license (http://creativecommons.org/licenses/by/4.0/). 